\title{
Südafrika: Kein Therapieeffekt bei latenter Tuberkulose
}

\author{
Die Tuberkulose (TB) gehört weiterhin zu den häufigsten \\ Infektionskrankheiten und Todesursachen weltweit. Gerade \\ in Südafrika stellen Koinfektionen mit TB und dem Humanen \\ Immundefizienz-Virus (HIV) ein Problem dar. Präventive \\ Ansätze sind entscheidend, um die Ausbreitung dieser \\ Erkrankung in Hochrisikoregionen und -populationen \\ einzuschränken. In einer randomisierten Studie haben G. J. \\ Churchyard et al. nun den Erfolg von präventiven Maßnahmen \\ am Beispiel von Minenarbeitern in Südafrika untersucht. \\ N Engl J Med 2014; 370: 301-310
}

In den 1960er Jahren wurde in Alaska, das zu der Zeit ein TB-Epidemiegebiet war, eine randomisierte Studie zum präventiven Einsatz von Isoniazid durchgeführt, welche deutliche Erfolge brachte. Davon inspiriert wurde nun an Minenarbeiter in Südafrika, welche eine Hochrisikopopulation darstellen, eine Studie zum Einsatz von Isoniazid durchgeführt. Im Gegensatz zur Studie aus Alaska zeigte die breitangelegte, 9-monatige Isoniazid-Prophylaxe hier jedoch keinen Effekt.

Weltweit nimmt die Inzidenz der TB zwar ab, dennoch bleibt sie, neben HIV und Malaria, eine der häufigsten Infektionskrankheiten und stellt vor allem in ärmeren Ländern ein substanzielles Problem dar. Die Weltgesundheitsorganisation (WHO) schätzt, dass rund ein Drittel der Weltbevölkerung mit TB infiziert ist. Die Erkrankung bricht jedoch nur bei 5-10\% der Betroffenen im Laufe des Lebens aus. Da TB gerade bei immungeschwächten Personen zu schweren Erkrankungsverläufen führt, ist die Kombination aus der chronisch-mykobakteriellen Infektion mit HIV/AIDS (und auch Mangelernährung), wie sie häufig in Afrika auftritt, sehr gefährlich.

Ein Drittel der jährlichen TB-Todesfälle entfällt auf Afrika, weshalb gerade hier therapeutische Strategien gesucht werden, um diese Erkrankung besser in den Griff zu bekommen. Die antibiotische Behandlung der TB wird erschwert durch eine monatelange Behandlungsdauer, welche eine entsprechende Compliance voraussetzt und gerade in wirtschaftlich schwachen Regionen oft schwer umsetzbar ist. Dennoch ist hier der medizinische Handlungsbedarf am größten. Daher haben die Wissenschaftler aus Johannesburg eine groß angelegte randomisierte Studie zur antibiotischen präventiven Therapie der TB

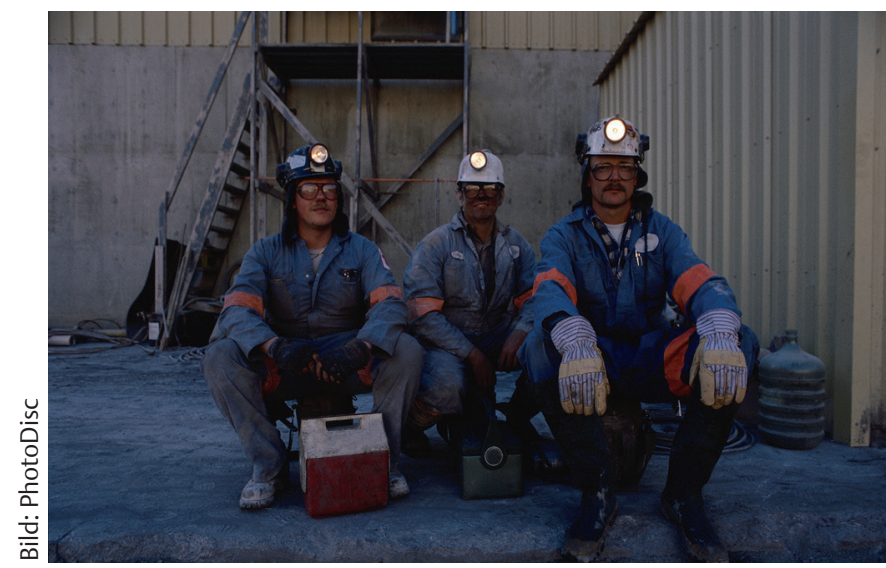

durchgeführt. In die Studie wurden initial 78744 Minenarbeiter eingeschlossen, welche nicht nur wegen HIV/AIDS, sondern auch wegen Silikose als TB-Hochrisikopopulation gelten. Nach einer Screeningphase wurden 23659 Arbeiter mit positivem Nachweis einer latenten oder aktiven TB mit Isoniazid behandelt. Die Behandlung hatte jedoch keinen statistisch signifikanten Einfluss auf die Inzidenz der TB im Beobachtungszeitraum.

\begin{abstract}
Fazit
Diese Studie steht im Kontrast zu früheren Untersuchungen, bei denen der breit angelegte Einsatz von Isoniazid zur Prävention der latenten Tuberkulose in Hochrisikopopulationen erfolgreich war. Weder Inzidenz, Prävalenz noch TB-assoziierte Mortalität wurden nach Angaben der Autoren durch die antibiotische Chemotherapie in der aktuellen Studie aus Südafrika reduziert. Die zugrunde liegenden Ursachen für das negative Studienergebnis bleiben weitgehend unklar.
\end{abstract}

\section{Prof. Dominik Hartl, Tübingen}

\section{Kommentar zur Studie}

Die vorliegende Studie umfasste eine hohe Fallzahl in einem Hochrisikogebiet, was die medizinische Versorgungsrelevanz dieser Fragstellung und Untersuchung unterstreicht. Relativ offen bleibt die Frage, welche Faktoren für den negativen Effekt der antibiotischen Intervention verantwortlich sind. Dies wird von den Autoren nur kurz diskutiert, stellt allerdings einen entscheidenden Punkt der Studie dar. Die Autoren spekulieren, dass durch den engen und kontinuierlichen Kontakt der Minenarbeiter untereinander, TB-Reaktivierungen, gerade nach Interventionsende, rasch auftreten könnten. Die Begleiterkrankungen AIDS und Silikose, die bei den untersuchten Minenarbeitern überdurchschnittlich häufig vorhanden waren, begünstigen zudem das Überleben der Mykobakterien in den Atemwegen der Betroffenen und schwächen den positiven Effekt der Isoniazidtherapie ab. Weitere Studien mit engeren Einschlusskriterien (z.B. weniger Komorbiditäten und -infektionen) sind notwendig, um die klinische Effektivität von präventiven IsoniazidInterventionen bei Patienten mit Tuberkulose genauer zu verstehen. 